ARTICLE

DOI: $10.1038 /$ s41467-017-00070-0

\title{
Sequential self-assembly of DNA functionalized droplets
}

Yin Zhang ${ }^{1}$, Angus McMullen', Lea-Laetitia Pontani ${ }^{1,3}$, Xiaojin He ${ }^{1}$, Ruojie Sha², Nadrian C. Seeman ${ }^{2}$, Jasna Brujic ${ }^{1} \&$ Paul M. Chaikin ${ }^{1}$

Complex structures and devices, both natural and manmade, are often constructed sequentially. From crystallization to embryogenesis, a nucleus or seed is formed and built upon. Sequential assembly allows for initiation, signaling, and logical programming, which are necessary for making enclosed, hierarchical structures. Although biology relies on such schemes, they have not been available in materials science. Here, we demonstrate programmed sequential self-assembly of DNA functionalized emulsions. The droplets are initially inert because the grafted DNA strands are pre-hybridized in pairs. Active strands on initiator droplets then displace one of the paired strands and thus release its complement, which in turn activates the next droplet in the sequence, akin to living polymerization. Our strategy provides time and logic control during the self-assembly process, and offers a new perspective on the synthesis of materials.

\footnotetext{
${ }^{1}$ Physics Department, Center for Soft Matter Research, New York University, 4 Washington Place, New York, New York 10003, USA. ${ }^{2}$ Chemistry Department, New York University, 100 Washington Square East, New York, New York 10003, USA. ${ }^{3}$ Present address: Institut des NanoSciences de Paris, UMR 7588, Centre National de la Recherche Scientifique-University Pierre et Marie Curie, 4 Place Jussieu, Paris, France. Correspondence and requests for materials should be addressed to N.C.S. (email: ned.seeman@nyu.edu) or to J.B. (email: jasna.brujic@nyu.edu) or to P.M.C. (email: chaikin@nyu.edu)
} 
O ver the past decade, there has been growing interest in using colloids for the self-assembly of arbitrarily designed structures ${ }^{1-4}$, rather than the more conventional colloidal crystals fabricated over the past century ${ }^{5-8}$. Colloidal particles with specific interparticle recognition facilitated by their functionalization with DNA sticky ends ${ }^{9-11}$, lock and key interactions ${ }^{12}$, as well as particles of different shapes and symmetrically arranged patches ${ }^{13}, 14$, have led to theoretical proposals for the creation of demonstrative target structures, e.g., a colloidal version of the Empire State building ${ }^{4}$. Such proposals rely on self-assembly through tunable enthalpic and entropic interactions guiding the system toward thermodynamic equilibrium. In addition, introducing mobile linkers to colloids ${ }^{15}$, liposomes ${ }^{16,17}$, and droplets ${ }^{18-20}$ avoids kinetic traps along the self-assembly pathways. However, the design principle that invokes sequential self or directed assembly has not yet been addressed due to the absence of an underlying technology. Sequential assembly is used industrially to make everything from integrated circuits to skyscrapers and is required in the growth of all living organisms. In many cases, it is a convenient organizational tool, in others, such as the three-dimensional enclosed structures of the Russian dolls, it is necessary to assemble the inside before the outside.

In this article, we demonstrate a technique for programmed self-assembly on the colloidal scale using the specific hybridization and easy design properties of DNA sequences ${ }^{21}$. Related schemes have been shown on molecular scales using DNA duplexes or DX motifs $^{22-24}$. In our system, a family of diversely functionalized emulsion droplets... B, C, D,... are self-assembled such that $\mathrm{C}$ only binds to $\mathrm{D}$ after $\mathrm{B}$ binds to $\mathrm{C}$. This process is controlled by using protected pairs of DNA strands on the droplets and a well-developed technology, toehold strand displacement ${ }^{25-27}$, as the enabling mechanism. With $\mathrm{A}$ as an initiator and $\mathrm{E}$ as a termination droplet, we spell out a droplet sequence ABCDE.

\section{Results}

Experimental design. In our design, we graft mobile DNA linkers to micro-scaled emulsion droplets, as depicted in Fig. 1a. In step 1, a set of droplets B and C have been prepared with pairs of DNA strands that are protected by their partial hybridization. In practice, each droplet has tens of thousands of such pairs. The protection renders the droplets inert-no bonds are formed between protected droplets. The complementary orange DNA sequences on $\mathrm{B}$ are hybridized and protected from the unpaired orange sequence on C. Similarly, the paired yellow sequences on $\mathrm{C}$ are protected from the unpaired yellow DNA strand on B. In step 2, an initiator droplet, $A$, is introduced. The DNA sequence on A has a section that is complementary to the unpaired red sequence and half of the paired orange sequence on B. Following the scheme of the toehold strand displacement mechanism (Fig. 1b), the complementary red sequences and half of the orange sequence bind by the DNA strand-branch migration. With proper sequence design, the remaining half of the originally hybridized yellow sequence is above its melting temperature and unbinds. Droplet A and B are then bound and the orange yellow DNA single strand is left unbound. If it were attached to a solid colloidal particle, it would be localized to the region near the $\mathrm{A}-\mathrm{B}$ binding site. However, on an emulsion droplet, it is free to diffuse over the entire droplet surface, as shown in step 3 of Fig. 1a. Droplet B is then activated and can serve as an initiator for binding and activation of droplet $\mathrm{C}$.

DNA functionalized droplets are different from similarly functionalized colloids in several ways related both to the mobility of the DNA ligands and the deformability of the droplets $^{28-31}$. When droplets approach, a few DNA strands on one droplet can hybridize with complementary strands on the other droplet making an initial bond. However, surface diffusion allows additional DNA strands to migrate to the contact region to form additional hybridized bonds and a

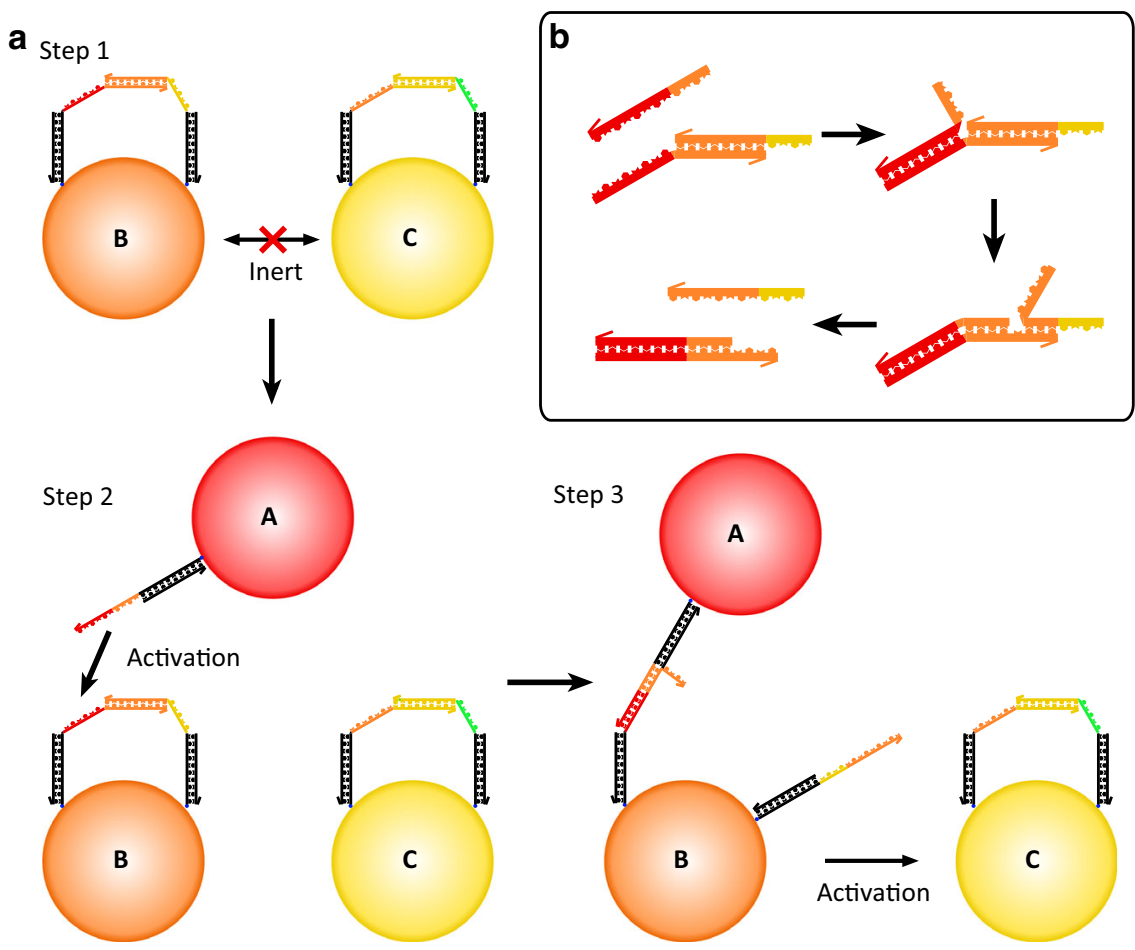

Fig. 1 A schematic of sequential self-assembly. a Step 1: two droplets, B and C, with partially hybridized pairs of DNA strands are inert. Step 2: a droplet with an initiator strand, A, is introduced. Step 3: the initiator strand fully hybridizes to the protection strand on $B$ through toehold strand displacement and frees the protected strand, leading to the activation of droplet $B$. The freed strand on $B$ can then hybridize with the protection strand on $C$ making a $B-C$ bond and activating $\mathrm{C}$ for further binding. $\mathbf{b}$ Schematic of the toehold displacement reaction showing details corresponding to the activation process of (a) 

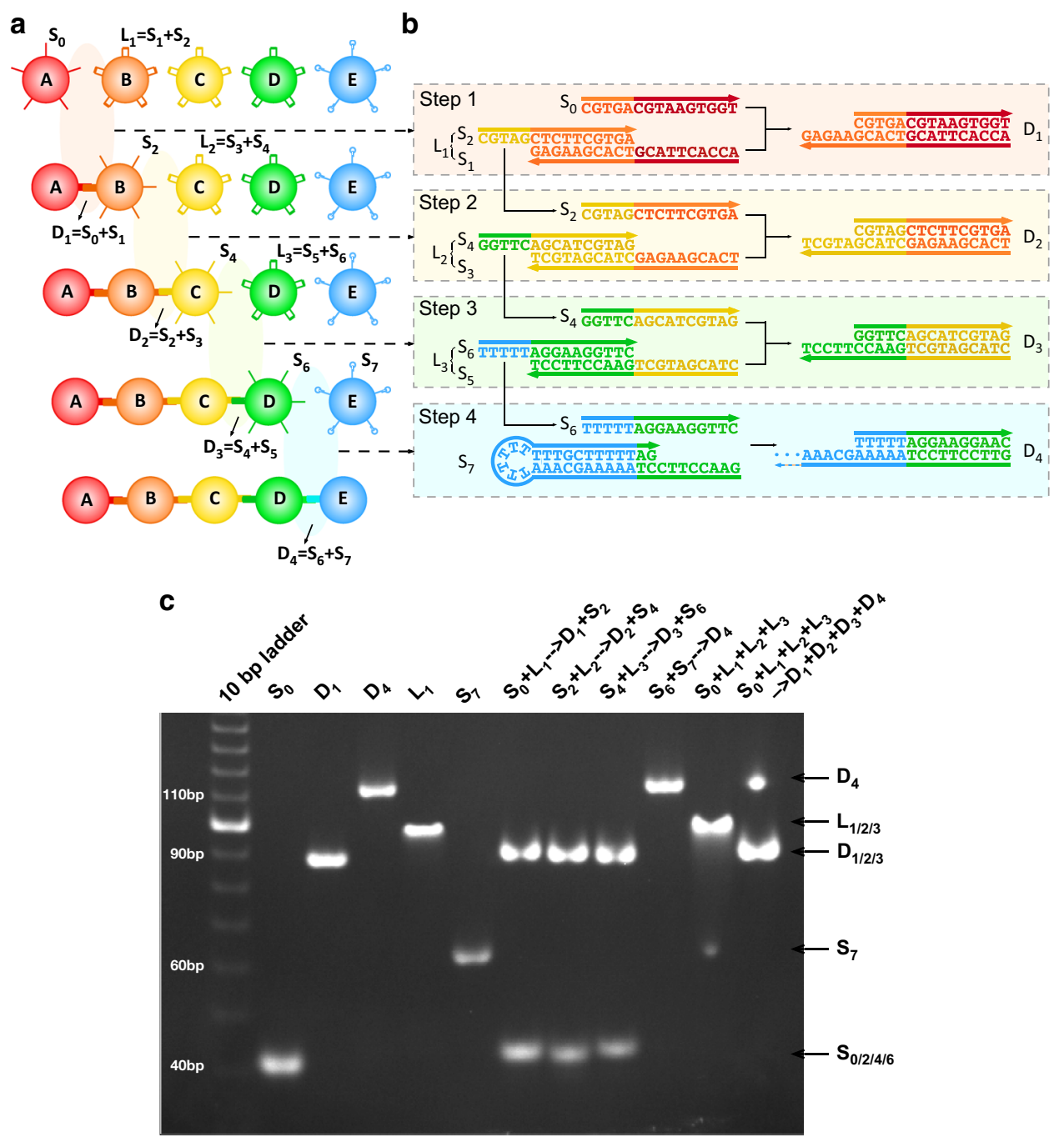

Fig. 2 Experimental design and gel results. a The programmed assembly sequences of the fluorescently labeled droplets used in this study. $\mathbf{b}$ The toehold strand displacement in each step corresponds to the sequential binding of droplets and their progressive activation of droplets in a. c Native PAGE demonstrates the step-by-step toehold strand displacement reactions depicted in $\mathbf{b}$. Column '10 bp ladder' is the marker lane. Column $\mathrm{S}_{0}$ serves as a reference for single strands $S_{2}, S_{4}$, and $S_{6}$ with the similar molecular weights. Similarly $D_{1}$ is a reference for $D_{2}$ and $D_{3}$ (completely hybridized duplex strands); $L_{1}$ is a reference for $L_{2}$ and $L_{3}$ (partially hybridized strands), while $S_{7}$ and $D_{4}$ mark the terminator hairpin strand and its partially hybridized duplex strands. Columns $S_{0}+L_{1} \rightarrow D_{1}+S_{2}, S_{2}+L_{2} \rightarrow D_{2}+S_{4}, S_{4}+L_{3} \rightarrow D_{3}+S_{6}$, and $S_{6}+S_{7} \rightarrow D_{4}$ show that the reactions depicted in $\mathbf{b}$ produce the desired results and leave none of the initial reactants. Column $L_{1}+L_{2}+L_{3}+S_{7}$ shows that the partially hybridized protected strand pairs are inert. The final column shows that the reactions go to completion when the initiator is added

growing patch. The patch flattens the interface between the droplets at the cost of elastic energy (surface tension). The balance between the hybridization energy and the elastic energy gives rise to an equilibrium patch size with a given number of strands, $N_{s}$. If the number of DNA strands on a droplet is less than or equal to the equilibrium $N_{s}$, then the droplet should be monovalent and binds to only one other droplet. In the present case, we use a low concentration of initiator strands and a low volume fraction of droplets to ensure that we are in the monovalent regime for each binding event. The number of released strands must be less than or equal to the number bound in each previous step in the sequence.

As a demonstration of sequential assembly, we choose a simple design - a set of droplets each labeled with a unique color. The designed sequence of events is shown in Fig. 2a. Droplet A is the initiator, while droplets $\mathrm{B}, \mathrm{C}, \mathrm{D}$ have protected pairs of DNA strands, and droplet $\mathrm{E}$ is a terminator with single DNA strand protected in a hairpin configuration. The sequences for the active regions of $\mathrm{A}-\mathrm{E}$ droplets are shown in Fig. $2 \mathrm{~b}$. Droplets $\mathrm{B}, \mathrm{C}, \mathrm{D}$, and $\mathrm{E}$ are prepared separately and then added to the same bath, since they are inert. When the initiator droplets A are added, we begin to see the sequential assembly of droplets binding, illustrated in Fig. 2a, and the corresponding strand displacement reactions in Fig. $2 b$. The system forms dimers, trimers, tetramers, and pentamers in the correct color order (and nothing longer).

Gel electrophoresis. Our sequential assembly scheme relies on the stability of the protected strand pairs and the effectiveness of strand displacement. These properties were tested by a series of experiments on the individual strands before attachment to the emulsion droplets. (Note that each single strand illustrated in Fig. $2 \mathrm{~b}$ is attached to a 36 nucleotide spacer strand, which can be hybridized to a complementary strand to make a duplex spacer with a biotinylated end allowing both attachment to a fluorescent streptavidin and separation of the active region from the droplet surface.) In Fig. 2c, we show a non-denaturing gel study verifying the protections and reactions between sequences depicted in 


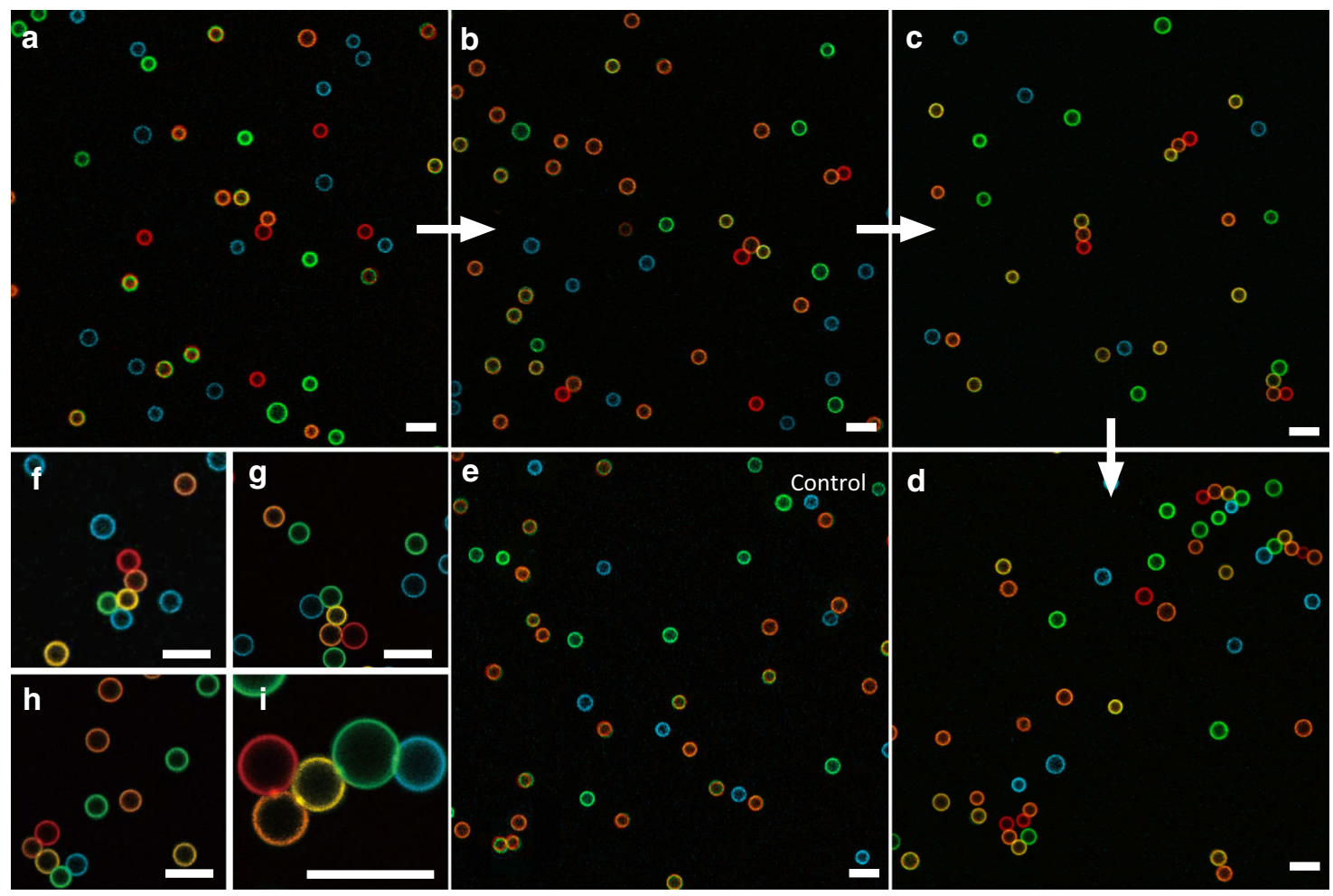

Fig. 3 Representative confocal images of sequentially self-assembled structures. In the experiment, droplets A, B, C, D, and E were false colored red, orange, yellow, green, and blue. a-d Images taken after $10 \mathrm{~h}, 26 \mathrm{~h}, 74 \mathrm{~h}$, and $101 \mathrm{~h}$ of incubation, respectively, showing the growth of triggered sequential self-assembly (SSA). e An image taken from the control experiment (no initiators) after 101-h incubation. f-h A gallery of various self-assembled pentamers. i A zoom-in view of a pentamer showing the patch formed at each droplets binding. All scale bars are $10 \mu \mathrm{m}$

Fig. 2b. Lane $S_{0}$ indicates the band for strand $S_{0}$ and serves as a reference for the single strands $S_{2}, S_{4}$, and $S_{6}$ with similar molecular weight. Similarly $D_{1}$ is a reference for bands $D_{2}$ and $\mathrm{D}_{3}$ (completely hybridized duplex strands), $\mathrm{L}_{1}$ is a reference for $\mathrm{L}_{2}$ and $\mathrm{L}_{3}$ (partially hybridized strands), while $\mathrm{S}_{7}$ and $\mathrm{D}_{4}$ mark the terminator hairpin strand and its partially hybridized duplex strands. Lanes $\mathrm{S}_{0}+\mathrm{L}_{1} \rightarrow \mathrm{D}_{1}+\mathrm{S}_{2}, \mathrm{~S}_{2}+\mathrm{L}_{2} \rightarrow$ $\mathrm{D}_{2}+\mathrm{S}_{4}, \mathrm{~S}_{4}+\mathrm{L}_{3} \rightarrow \mathrm{D}_{3}+\mathrm{S}_{6}$, and $\mathrm{S}_{6}+\mathrm{S}_{7} \rightarrow \mathrm{D}_{4}$ show that the reactions depicted in Fig. $2 \mathrm{~b}$ produce the desired results and leave none of the initial reactants. Lane $\mathrm{L}_{1}+\mathrm{L}_{2}+\mathrm{L}_{3}+\mathrm{S}_{7}$ shows that the partially hybridized protected strand pairs do not react in the absence of the initiator. On the other hand, the final lane shows that when the initiator strand is added to the previously inert protected strands, the complete set of reactions goes to completion with only complete duplex strands and the partially hybridized termination duplex strands as the result.

DNA functionalization. Having confirmed the success of the molecular scheme, we next functionalize the droplets to drive their polymerization. The experiments are performed with micron-sized, lipids stabilized silicone oil in water emulsion droplets (see methods). DNA loops $\left(\mathrm{L}_{1}, \mathrm{~L}_{2}\right.$, and $\left.\mathrm{L}_{3}\right)$ are pre-annealed and purified from native gels to ensure the correct stoichiometry. A 'biotinylated phospholipid-fluorescent streptavidin-biotinylated oligonucleotide' linking strategy is applied to functionalize the oil droplets with the corresponding DNA structures $\left(\mathrm{S}_{0}, \mathrm{~L}_{1}, \mathrm{~L}_{2}, \mathrm{~L}_{3}\right.$, and $\left.\mathrm{S}_{7}\right)$. In the experiment, each combination of streptavidin with different fluorescent labels is specifically associated with one type of DNA strand/loop structure to distinguish them using fluorescence confocal microscopy. From fluorescence spectroscopy measurements, we estimate that the surface coverage of streptavidin on droplets is $\sim 1000 \mu \mathrm{m}^{-2}$ (Supplementary Methods and Supplementary Fig. 3).

Confocal imaging and data analysis. After DNA functionalization, the droplets are mixed together at number ratio of $A: B: C$ : $\mathrm{D}: \mathrm{E}=1: 2: 2: 2: 2$, and loaded into a homemade sample chamber (Supplementary Methods). The droplets cream to the top surface forming a monolayer, and diffuse thermally. The chamber was placed on a leveled surface in an incubator at $12^{\circ} \mathrm{C}$. The sample is imaged using fluorescence confocal microscopy (Leica TCS SP5 II) at different time points. Confocal images demonstrate the growth of sequential clusters with initiators (Fig. 3a-d and Supplementary Figs. 3-7) and a control without initiator (Fig. 3e). The control shows little aggregation after $101 \mathrm{~h}$ with $\sim 87 \%$ of the droplets staying unbound. The experiments with initiators show the droplets first self-assemble into dimers, then trimers, tetramers, and finally pentamers (Fig. $3 \mathrm{f}-\mathrm{i}$ ). Figure 4 quantifies these results showing that, in the presence of the initiators, by $101 \mathrm{~h}$ the dominant population (35\% of the initiators) is the programmed pentamer in the correct sequence, and $94 \%$ of the initiators have been used up. Another analysis (Fig. 4 inset) indicates that initiated sequential bindings formed in the system are six times more than the number of mistakes (uninitiated bindings or bindings in the wrong sequence). Among the initiated sequential bindings, $73 \%$ are in linear regime (only one predecessor and one successor) and $27 \%$ in branched regime (multiple predecessors or successors). At present, the control of monovalent binding is less than perfect. Reducing the DNA surface density on droplets will further optimize our system, leading to a higher yield of linear chains. 


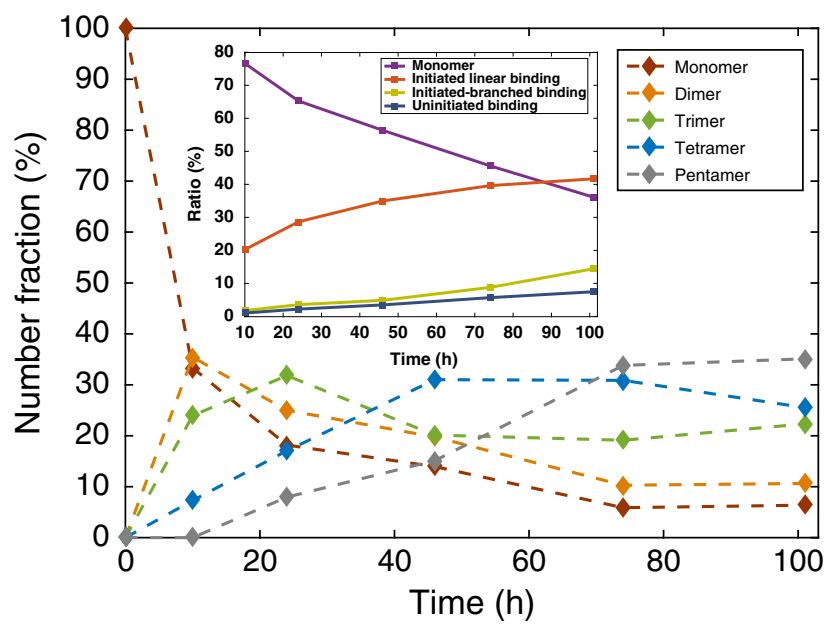

Fig. 4 The evolution of the distribution of assembled chains of various lengths. Each data point is the ratio of the number of initiators in chains of a certain length to the total number of initiators from the analysis (see Supplementary Tables 1 and 2 for the counting results). Each curve demonstrates the change of the number fraction of the assembled structures of a certain length with time. This group of curves presents the majority of the assembled structures in the system progress from monomers to dimers, trimers, tetramers, and finally pentamers. The inset shows the proportions of droplets staying as unbound monomers, with initiated linear (only one predecessor and one successor) and branched (multiple predecessors or successors) bindings, as well as non-specific bindings at different time points. The droplets with non-specific bindings account for the ones in wrong sequences (e.g., $A-C, B-E$ or $D-D$, etc.) or uninitiated assemblies (e.g., $B-C$ or $C-D-E$, etc.)

Kinetics model for the sequential self-assembly. The time scale for these reactions is governed by the aggregation kinetics of monolayer DNA-coated droplets on a surface as studied in ref. ${ }^{32}$. The aggregation time $\tau$ can be obtained from:

$$
\tau=\tau_{\mathrm{DLA}}+\frac{\tau_{\mathrm{R}}}{4 \pi R_{\mathrm{d}} L C_{0}},
$$

where

$$
\tau_{\mathrm{DLA}}=\frac{-\ln \left(4 \pi e R_{\mathrm{d}}^{2} C_{0}\right)-1}{8 \pi D_{0} C_{0}}
$$

$\tau_{\mathrm{R}}$ is the reaction time of a pair of droplets with complementary sticky-end DNA on their surfaces staying within the reaction region (thickness $L \sim 10 \mathrm{~nm}$ ). $C_{0}$ is the number density of droplets of one particular type, $\sim 3 \times 10^{2} \mathrm{~mm}^{-2}$ for droplet $\mathrm{A}$ and $\sim 6 \times 10^{2} \mathrm{~mm}^{-2}$ for droplets $\mathrm{B}, \mathrm{C}, \mathrm{D}$, and E. $R_{\mathrm{d}}$ is the average radius of the emulsion droplets, $\sim 2.35 \mu \mathrm{m} ; D_{0}$ is the measured two dimensional diffusion coefficient of droplets, $\sim 0.037 \mu \mathrm{m}^{2} \mathrm{~s}^{-1}$. Our system is reaction limited with $\tau_{\text {DLA }} \sim 0.5 \mathrm{~h}$ and the rate of DNA-coated droplet binding (the second term in the right hand side of Equation 1) $\sim 0.2-2 \mathrm{~h}$ for the initiator reaction and $\sim 2-20 \mathrm{~h}$ for the subsequent reactions (Supplementary Note 1). For hybridization to occur, the accumulated contact time for a pair of strands to be hybridized is $\sim 1 \mathrm{~s}$. Strands diffuse on the droplet surface quickly and make contact many times before binding from one droplet to another. Thus, $\tau_{\mathrm{R}}$ depends on the diffusion coefficient and density of active DNA on the droplet surface. For the initiator, the number of active strands is measured by fluorescence to be $\sim 12,000$, corresponding to a strand density $\sim 200$ per $\mu \mathrm{m}^{2}$. The kinetic model allows us to estimate the number of released strands from the adhesion patch to be $\sim$ 1200 or $20 \mu^{-2}$ in order to match the observed assembly time scales demonstrated in Fig. 4. To test the robustness of our system, the experiment is carried out using another batch of smaller droplets $(3.4 \mu \mathrm{m})$ with higher diffusion coefficient $\left(0.08 \mu \mathrm{m}^{2} \mathrm{~s}^{-1}\right)$. The results present a similar yield (Supplementary Tables 3 and 4) and assembly kinetics (Supplementary Fig. 8), which have also been confirmed by theoretical calculation (Supplementary Note 1).

\section{Discussion}

We have demonstrated a technique and developed a simple system to allow for the sequential assembly of micron-scale objects. The experimental parameters, such as temperature, DNA sequences, droplet density, size, and grafting chemistry can be optimized for faster growth, error reduction or increasing/ decreasing branching. For instance, we can use a longer duplex in the loop structures for added stabilization, which should minimize uninitiated binding. We could also improve our yield through magnetic or surface tethered initiators, allowing us to remove the unreacted monomers and non-specific clusters. This method can be further extended in many directions. Programmed sequential self-assembly can produce stable non-equilibrium structures similar to the directed sequential growth achieved by molecular beam epitaxy ${ }^{33}$. A cyclic system, IABCABCABC... where the released strand on $C$ is the same as the initiator strand would allow for an extended structure and a true emulsion analog to living polymerization. The linear chains can be folded into two or three-dimensional structures by coating surfaces with several different species of DNA, which can later be turned on chemically or by cooling. An initiator or subsequently freed strand can bind to release more than one type of strand in the next sequence step (by using the same protection strand on two partially different sequences or protecting two strands with one). This would allow control of branching and/or the direct construction of three-dimensional objects. Conversely, two or more initiator strands could be required to bind to and activate a droplet. Lipid coatings on colloidal particles enable mobility on their surfaces ${ }^{15}$, and thus serve as an extension of our technique to solid particles. Logic and sequence control should greatly expand the range of structures and devices accessible to colloidal/emulsion self-assembly.

\section{Methods}

Droplet fabrication. In the experiment two batches of droplets with different sizes were used. They were made via different methods. The $4.7 \mu \mathrm{m}$ droplets: $5 \mathrm{ml}$ of silicone oil (viscosity $=50 \mathrm{cst}$, from Sigma Aldrich) was extruded through a membrane with a mean pore size of $1 \mu \mathrm{m}$ using a membrane emulsification device (internal pressure type micro kit, SPG Technology Co., Ltd). The droplets were stored in a $10 \mathrm{mM}$ sodium dodecyl sulfate (SDS) buffer at $4{ }^{\circ} \mathrm{C}$ after two cycles of washes. The average diameter was $4.7 \mu \mathrm{m}$. The $3.4 \mu \mathrm{m}$ droplets $^{34}: 5 \mathrm{ml}$ of DMDES was vortexed with $500 \mu \mathrm{l}$ of deionized water for $5 \mathrm{~min}$, then left on a rotator for $30 \mathrm{~min}$ until the mixture was homogeneous. We then mixed a solution of $6 \% \mathrm{v} / \mathrm{v}$ prehydrolyzed DMDES and $20 \% \mathrm{v} / \mathrm{v}$ ammonia (total volume was $35 \mathrm{ml}$ ), and vortexed for $5 \mathrm{~min}$. The droplets were left to grow undisturbed for $24 \mathrm{~h}$, until the droplets were monodisperse and $\sim 1.75 \mu \mathrm{m}$ in diameter. After growth, we dialyzed the droplet solution against a $1 \mathrm{mM}$ SDS solution until all traces of ammonia were removed. We then restarted the reaction by adding $1.5 \mathrm{ml}$ of prehydrolyzed DMDES and $1 \% \mathrm{v} / \mathrm{v}$ ammonia. The PDMS droplet seeds were left to grow undisturbed until their growth had saturated. More prehydrolyzed DMDES was added until the droplets had reached the desired diameter. These droplets were again dialyzed against a $1 \mathrm{mM}$ SDS solution until all unreacted monomer and ammonia were removed, and then stored in a $10 \mathrm{mM}$ SDS buffer at $4{ }^{\circ} \mathrm{C}$ until the droplets were ready to be labeled with lipids.

Phospholipids labeling. We used different methods to functionalize the two batches of droplets with phospholipids. For the $4.7 \mu \mathrm{m}$ droplets: egg L- $\alpha$-phosphatidylcholine lipids and DSPE-PEG (2000) biotin (both from Avanti Polar Lipids) were mixed at a molar ratio of 95:5, and evaporated under filtered nitrogen. The dried phospholipids were then dissolved in dimethyl sulfoxide (Sigma Aldrich) to $100 \mathrm{gl}^{-1}$, and further diluted in a $10 \mathrm{mM}$ Tris- $\mathrm{HCl}, \mathrm{pH} 8,1 \mathrm{mM}$ SDS, $0.1 \mathrm{mM}$ $\mathrm{NaN}_{3}$ buffer to $1 \mathrm{gl}^{-1}$. The lipids buffer was then added to the creamed emulsion 
droplets at a volume ratio of $1000 \mu \mathrm{l}: 100 \mu \mathrm{l}$. Then the mixture was diluted to $10 \mathrm{ml}$ using the same buffer, and tumbled gently at room temperature overnight. The emulsion was washed three times using the TMS buffer $(10 \mathrm{mM}$ Tris- $\mathrm{HCl}, \mathrm{pH} 8$, $3 \mathrm{mM} \mathrm{MgCl}_{2}, 1 \mathrm{mM}$ SDS, $0.1 \mathrm{mM} \mathrm{NaN}_{3}$ ) and then stored at $4{ }^{\circ} \mathrm{C}$ for further use. These emulsions are stable for several months. For the $3.4 \mu \mathrm{m}$ droplets: DSPE-PEG (2000) (1,2-distearoyl-sn-glycero-3-phosphoethanolamine- $\mathrm{N}$-[amino(polyethylene glycol)-2000]) and DSPE-PEG (2000) biotin (both dissolved in chloroform) were mixed at a 20:1 molar ratio. Approximately $10 \mu \mathrm{l}$ of this lipid mixture $\left(10 \mathrm{gl}^{-1}\right.$ in chloroform) was added to a clean glass vial. We then evaporated off all chloroform with nitrogen, and resuspended the lipid cake in $70 \mu$ l of dimethyl sulfoxide (Sigma Aldrich). To this mixture, we added $1 \mathrm{ml}$ of $10 \mathrm{mM}$ sodium acetate buffer (pH 5), $1 \mathrm{mM}$ SDS, $1 \mathrm{mM}$ EDTA. Then, $50 \mu \mathrm{l}$ of creamed emulsion droplets were added in this solution, and left to incubate on a rotator for $24 \mathrm{~h}$. The droplets were then washed three times in a $10 \mathrm{mM}$ Tris- $\mathrm{HCl} \mathrm{pH} 8,1 \mathrm{mM}$ Tergitol TMN-6, $1 \mathrm{mM}$ EDTA buffer to remove any unattached lipids. Lipid-functionalized droplets were stored at $4{ }^{\circ} \mathrm{C}$ until ready to be complexed with streptavidin and DNA.

DNA sequence. All the sequences were generated using the program Uniquimer and were purchased from Integrated DNA Technology, Inc (www.idtdna.com). The biotinylated CS strands (Btn-CS) were HPLC purified. The strands $S_{0}-S_{7}$ and PolyT were purified by denaturing PAGE. Below are the sequences:

Btn-CS: CATCGAACAATCCGGTCGAGTGCCATGATTTGTGAG/3BioTEG/ PolyT: CTCACAAATCATGGCACTCGACCGGATTGTTCGATGTTTT TTTTTTT

$\mathrm{S}_{0}$ : CTCACAAATCATGGCACTCGACCGGATTGTTCGATGCGTGACG TAAGTGGT

$\mathrm{S}_{1}$ : CTCACAAATCATGGCACTCGACCGGATTGTTCGATGACCACT TACGTCACGAAGAG

$\mathrm{S}_{2}$ : CTCACAAATCATGGCACTCGACCGGATTGTTCGATGCGTAGCTC TTCGTGA

$\mathrm{S}_{3}$ : CTCACAAATCATGGCACTCGACCGGATTGTTCGATGTCACGAAGA GCTACGATGCT

$S_{4}$ : CTCACAAATCATGGCACTCGACCGGATTGTTCGATGGGTTCAG

CATCGTAG

$S_{5}:$ CTCACAAATCATGGCACTCGACCGGATTGTTCGATGCTACG ATGCTGAACCTTCCT

$\mathrm{S}_{6}$ : CTCACAAATCATGGCACTCGACCGGATTGTTCGATGTTTTTA GGAAGGTTC

$S_{7}:$ CTCACAAATCATGGCACTCGACCGGATTGTTCGATGGAACCTTC CTAAAAAGCAAATTTTTTTTTGCTTTTTAG

Native polyacrylamide gel electrophoresis. The gel demonstrating results of the toehold strand displacement reactions was a $10 \%$ native gel. It was prepared in $1 \times \mathrm{TAE} / \mathrm{Mg}^{++}$buffer $\left(20 \mathrm{mM}\right.$ Tris-HCl, pH 7.5, $2 \mathrm{mM}$ EDTA, $6.25 \mathrm{mM} \mathrm{Mg}{ }^{++}$, $\mathrm{pH}=7.6$ ). The DNA structures were annealed following a standard protocol: heating at $95^{\circ} \mathrm{C}$ for $5 \mathrm{~min}$ followed by cooling to room temperature over $2.5 \mathrm{~h}$. The gel was run on a Hoefer SE-600 gel electrophoresis unit at $7 \mathrm{~V} \mathrm{~cm}^{-1}$ for $5 \mathrm{~h}$ at $12^{\circ} \mathrm{C}$, post-stained with $0.5 \mu \mathrm{g} \mathrm{ml}^{-1}$ ethidium bromide, and visualized by UV transillumination.

DNA functionalization. Overall, 50 pmol DNA loop structures $\mathrm{L}_{1}, \mathrm{~L}_{2}$, and $\mathrm{L}_{3}$ (native gel-purified), $100 \mathrm{pmol}$ initiator strand $\mathrm{S}_{0}$ and $100 \mathrm{pmol}$ hairpin strand $\mathrm{S}_{7}$ were separately annealed with an equimolar amount of biotinylated complementary strands in the magnesium buffer (TMS buffer for the strands functionalized on $4.7 \mu \mathrm{m}$ droplets; TMT buffer: $10 \mathrm{mM}$ Tris- $\mathrm{HCl} \mathrm{pH} 8,4 \mathrm{mM}$ $\mathrm{MgCl}_{2}, 1 \mathrm{mM}$ Tergitol TMN-6, $0.1 \mathrm{mM} \mathrm{NaN}_{3}$ for the strands functionalized on $3.2 \mu \mathrm{m}$ droplets, same in the following steps). Then different combinations of fluorescent streptavidin were added to the DNA solutions at a ratio of 1 streptavidin molecule: 1 biotin group. For the streptavidin added to $S_{0}: 100 \%$ were labeled with Alexa ${ }^{\circledR}$ Fluor $647 ; \mathrm{L}_{1}$ : 67\% Alexa ${ }^{\circledR}$ Fluor 647 and $33 \%$ Alexa ${ }^{\circledR}$ Fluor $488 ; \mathrm{L}_{2}$ : $33 \%$ Alexa ${ }^{\circledR}$ Fluor 647 and $67 \%$ Alexa ${ }^{\circledR}$ Fluor $488 ; \mathrm{L}_{3}: 100 \%$ Alexa ${ }^{\circledR}$ Fluor $488 ; \mathrm{S}_{7}$ : $100 \%$ Alexa ${ }^{\circledR}$ Fluor 546 . Meanwhile, $35 \mu \mathrm{l}(25 \mu \mathrm{l})$ of the creamed $4.7 \mu \mathrm{m}(3.4 \mu \mathrm{m})$ emulsion droplets were diluted in a $500 \mu \mathrm{l}$ magnesium buffer, and then split evenly into five parts. After a 30-min incubation, each DNA/streptavidin solution was mixed with one part of the emulsions. The mixtures were incubated for $1 \mathrm{~h}$ allowing for the binding of streptavidin to biotin groups on the emulsion droplet's surface. Then they were washed four times to remove the remaining unbound DNA and excess streptavidin. Each type of droplets were diluted using the same magnesium buffer to a final concentration $\sim 3 \times 10^{3} \mu^{-1}$.

Confocal fluorescent microscopy. Leica DM6000 CS confocal microscope (TCS SP5 II) was used to take fluorescent images of our samples. Excitations with $488 \mathrm{~nm}, 543 \mathrm{~nm}$, and $633 \mathrm{~nm}$ were used for imaging the samples. A $\times 63$ oil immersion objective (Leica) was used for all fluorescent imaging.

Data Availability. The data that support the findings of this study are available from the corresponding author on request.
Received: 17 April 2017 Accepted: 28 April 2017

Published online: 16 June 2017

\section{References}

1. Whitesides, G. M. \& Grzybowski, B. Self-assembly at all scales. Science $\mathbf{2 9 5}$, 2418-2421 (2002).

2. Whitesides, G. M. \& Boncheva, M. Beyond molecules: self-assembly of mesoscopic and macroscopic components. Proc. Natl Acad. Sci. USA 99, 4769-4774 (2002).

3. Szwarc, M. 'Living' polymers. Nature 178, 1168-1169 (1956).

4. Halverson, J. D. \& Tkachenko, A. V. DNA-programmed mesoscopic architecture. Phys. Rev. E 87, 062310 (2013).

5. Pieranski, P. Colloidal crystals. Contemp. Phys. 24, 25-73 (1983).

6. Leunissen, M. E. et al. Ionic colloidal crystals of oppositely charged particles. Nature 437, 235-240 (2005).

7. Kim, A. J., Biancaniello, P. L. \& Crocker, J. C. Engineering DNA-mediated colloidal crystallization. Langmuir 22, 1991-2001 (2006).

8. Wang, Y. et al. Crystallization of DNA-coated colloids. Nat. Commun. 6, 7253 (2015).

9. Mirkin, C. A., Letsinger, R. L., Mucic, R. C. \& Storhoff, J. J. A DNA-based method for rationally assembling nanoparticles into macroscopic materials. Nature 382, 607-609 (1996).

10. Valignat, M. P., Theodoly, O., Crocker, J. C., Russel, W. B. \& Chaikin, P. M. Reversible self-assembly and directed assembly of DNA-linked micrometersized colloids. Proc. Natl Acad. Sci. USA 102, 4225-4229 (2005).

11. Rogers, W. B. \& Manoharan, V. N. Programming colloidal phase transitions with DNA strand displacement. Science 347, 639-642 (2015).

12. Sacanna, S., Irvine, W. T. M., Chaikin, P. M. \& Pine, D. J. Lock and key colloids Nature 464, 575-578 (2010).

13. Wang, Y. et al. Colloids with valence and specific directional bonding. Nature 491, 51-55 (2012).

14. Chen, Q., Bae, S. C. \& Granick, S. Directed self-assembly of a colloidal kagome lattice. Nature 469, 381-384 (2011).

15. van der Meulen, S. A. J. \& Leunissen, M. E. Solid colloids with surface-mobile DNA linkers. J. Am. Chem. Soc. 135, 15129-15134 (2013).

16. Hadorn, M. \& Hotz, P. E. DNA-mediated self-assembly of artificial vesicles. PLoS ONE 5, E9886 (2010).

17. Parolini, L., Kotar, J., Di Michele, L. \& Mognetti, B. M. Controlling selfassembly kinetics of DNA-functionalized liposomes using Toehold exchange mechanism. ACS Nano 10, 2392-2398 (2016).

18. Hadorn, M. et al. Specific and reversible DNA-directed self-assembly of oil-inwater emulsion droplets. Proc. Natl Acad. Sci. USA 109, 20320-20325 (2012).

19. Feng, L., Pontani, L.-L., Dreyfus, R., Chaikin, P. M. \& Brujic, J. Specificity, flexibility and valence of DNA bonds guide emulsion architecture. Soft Matter 9, 9816-9823 (2013).

20. Joshi, D. et al. Kinetic control of the coverage of oil droplets by DNAfunctionalized colloids. Sci. Adv. 2, E1600881 (2016).

21. Seeman, N. C. DNA in a material world. Nature 421, 427-431 (2003).

22. Dirks, R. M. \& Pierce, N. A. Triggered amplification by hybridization chain reaction. Proc. Natl Acad. Sci. USA 101, 15275-15278 (2008).

23. Yin, P., Choi, H. M., Calvert, C. R. \& Pierce, N. A. Programming biomolecular self-assembly pathways. Nature. 451, 318-322 (2008).

24. Padilla, J. E. et al. A signal-passing DNA-strand-exchange mechanism for active self-assembly of DNA nanostructures. Angew. Chem. Int. Ed. 54, 5939-5942 (2015).

25. Yurke, B., Turberfield, A. J., Mills, A. P., Simmel, F. C. \& Neumann, J. L. A DNA-fuelled molecular machine made of DNA. Nature 406, 605-608 (2000).

26. Zhang, D. Y. \& Winfree, E. Control of DNA strand displacement Kinetics using toehold exchange. J. Am. Chem. Soc. 131, 17303-17314 (2009).

27. Srinivas, N. et al. On the biophysics and kinetics of toehold-mediated DNA strand displacement. Nucleic Acids Res. 41, 10641-10658 (2013).

28. Pontani, L. L., Jorjadze, I., Viasnoff, V. \& Brujic, J. Biomimetic emulsions reveal the effect of mechanical forces on cell-cell adhesion. Proc. Natl Acad. Sci. USA 109, 9839-9844 (2012).

29. Thiam, A. R., Farese, R. V. Jr \& Walther, T. C. The biophysics and cell biology of lipid droplets. Nat. Rev. Mol. Cell Biol. 14, 775-786 (2013).

30. Campàs, O. et al. Quantifying cell-generated mechanical forces within living embryonic tissues. Nat. Methods 11, 183-189 (2014).

31. Pontani, L. L., Haase, M. F., Raczkowska, I. \& Brujic, J. Immiscible lipids control the morphology of patchy emulsions. Soft Matter 9, 7150-7157 (2013).

32. Wu, K.-T. et al. Kinetics of DNA-coated sticky particles. Phys. Rev. E $\mathbf{8 8}$, 022304-022308 (2013).

33. Arthur, J. R. Molecular beam epitaxy. Surf. Sci. 500, 189-217 (2002). 
34. Elbers, N. A., Jose, J., Imhof, A. \& van Blaaderen, A. Bulk scale synthesis of monodisperse PDMS droplets above $3 \mu \mathrm{m}$ and their encapsulation by elastic shells. Chem. Mater. 27, 1709-1719 (2015).

\section{Acknowledgements}

This research has been primarily supported by DOE DE-SC0007991 to P.M.C., N.C.S., R.S., and X.H. for initiation, design, analysis, and imaging. We acknowledge partial support from the Gordon and Betty Moore Foundation to P.M.C., N.C.S., R.S., and X.H. for DNA sequence design, preparation, and characterization; grants EFRI-1332411 and CCF-1526650 from the NSF, MURI W911NF-11-1-0024 from ARO and N000141110729 from ONR to N.C.S. and R.S. for data analysis. Y.Z. acknowledges support from DOE CBES for sample preparation, confocal microscopy, and data analysis. A.M., L.-L.P.,

X.H., and J.B. acknowledge partial support from the MRSEC program of the National Science Foundation under Award Number DMR-1420073 for synthesis and characterization of the emulsion droplets. We are grateful for shared facilities provided through the MRSEC program of the National Science Foundation under Award Number DMR-1420073. We thank Wenhai Zheng for the measurement of the droplets diffusion coefficient.

\section{Author contributions}

Y.Z. and P.M.C. designed research; Y.Z., A.M., L.-L. P., X.H., and R.S. performed research; Y.Z. analyzed data; Y.Z., N.C.S., J.B., and P.M.C. wrote the paper.

\section{Additional information}

Supplementary Information accompanies this paper at doi:10.1038/s41467-017-00070-0.

Competing interests: The authors declare no competing financial interests.

Reprints and permission information is available online at http://npg.nature.com/ reprintsandpermissions/

Publisher's note: Springer Nature remains neutral with regard to jurisdictional claims in published maps and institutional affiliations.

(c) (i) Open Access This article is licensed under a Creative Commons Attribution 4.0 International License, which permits use, sharing, adaptation, distribution and reproduction in any medium or format, as long as you give appropriate credit to the original author(s) and the source, provide a link to the Creative Commons license, and indicate if changes were made. The images or other third party material in this article are included in the article's Creative Commons license, unless indicated otherwise in a credit line to the material. If material is not included in the article's Creative Commons license and your intended use is not permitted by statutory regulation or exceeds the permitted use, you will need to obtain permission directly from the copyright holder. To view a copy of this license, visit http://creativecommons.org/ licenses/by/4.0/.

(C) The Author(s) 2017 